\title{
Grid Reconstruction and Skew Angle Estimation in Integral Images Produced using Circular Microlenses
}

\author{
E. T. Koufogiannis ${ }^{1}$ N. P. Sgouros ${ }^{1}$ M. T. Ntasi ${ }^{1}$ M. S. Sangriotis ${ }^{1}$ \\ ${ }^{1}$ Department of Informatics and Telecommunications University of Athens, \\ Panepistimiopolis Athens, Greece 15784 \\ \{efthimis,nsg,sagri\}@di.uoa.gr
}

\begin{abstract}
Nowadays a number of different three-dimensional (3D) systems compete in the field of capturing and delivering autostereoscopic (ASt) 3D content. Integral Imaging (InI) is a promising ASt technique that provides both horizontal and vertical parallax as well as high quality realistic 3D content. In this work we propose an InI preprocessing method for identification and accurate segmentation of the grid structure in Integral Images (InIms) generated using lens arrays (LAs) containing circular lenses. In the proposed method we utilize the gradient augmented circular hough transform to accurately detect circular regions in the acquired integral image (InIm). Subsequently by using a triangulation scheme followed by a statistical approach we accurately estimate the grid line structure of the utilized LA. This results in the accurate segmentation of the circular shaped elemental images (EIs) contained in the InIm, a process vital for the effectiveness of the InI methodology. We provide experimental results over artificial as well as optically acquired InIms to evaluate the accuracy of the method using objective metrics.
\end{abstract}

\section{INTRODUCTION}

In recent years ASt imaging is quickly gaining ground due to the increased demand for glasses-free 3D content as well as the rapid development in display and image acquisition technologies. Amongst available ASt methods InI is a promising technique that was initially formulated by the Nobel laureate G. Lippman back in 1908 [1]. In the recent years it has attracted great users interest due to the ability of providing simultaneous horizontal and vertical (2D) parallax along with full color, adequate resolution, and support for multiple simultaneous viewers.

The setup utilized for capturing InIms is shown in Fig. 1(a) and it is assembled from a charged coupled device (CCD) and a lens array (LA) [2] [3]. The corresponding Ast display setup based on the InI technique is shown in Fig. 1(b) and consists of a liquid crystal display (LCD) and a LA.

During the capturing stage, an LA is placed between the CCD and the photographed object, and an elemental image (EI) is formed, on the CCD plane, behind each one of the lenses comprising the LA. The InIm formed on the CCD plane during this stage is subsequently projected during display through an appropriate LA as described above. This process

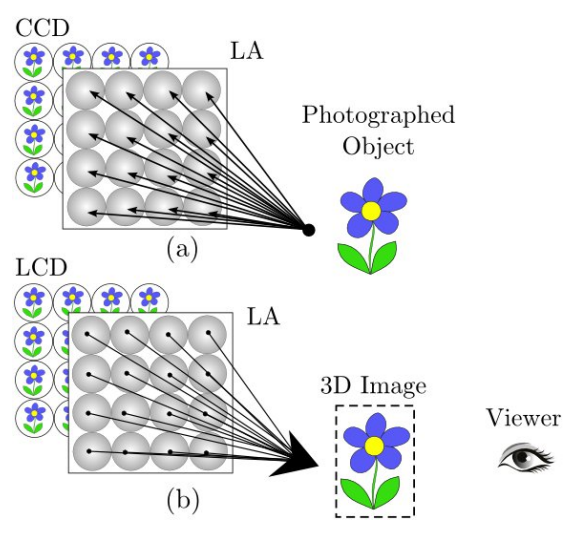

Fig. 1. (a) Integral Imaging acquisition, (b) InI display setup.

results in a realistic 3D object that appears floating between the viewer and the LA.

Existing LA configurations are characterized by various lens packing topologies as well as lens shapes. The currently existing InI setups are shown in Fig. 2 where we observe that different regular lens shapes can be utilized such as circular, square, hexagonal and triangular. Furthermore the lens packing topology can be either square or hexagonal, an approach aimed to improving the percentage of the utilized CCD surface.

An important problem during the capturing procedure is the existence of slight misalignment between the LA and the CCD planes. This misalignment introduces distortions that alter the expected position of the EIs in the acquired InIm. Additional defects in the LA surface results in slight lens size variability and this has the side effect of producing EIs that feature variation in size.

In an InI based display setup that assumes accurate and exact dimensions as well as correct positioning of the received EIs these errors propagate and resut in degrading the $3 \mathrm{D}$ effect, or completely destroying the projected 3D image. Furthermore since $3 \mathrm{D}$ reconstruction of the initially photographed object [4] as well as 2D view rendering and refocusing [5] and InIm compression [6] methodologies rely on accurate positioning of the EIs it is obvious that a preprocessing stage is required 


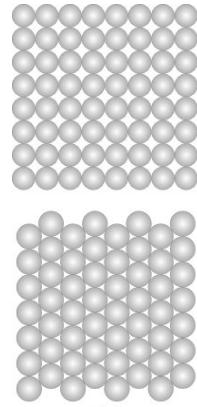

(a)
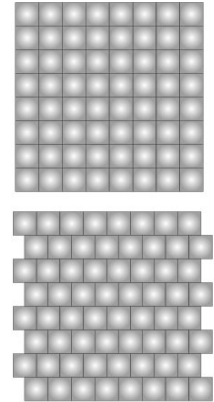

(b)
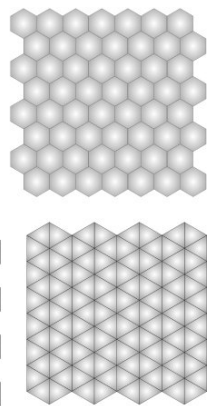

(c)
Fig. 2. Various LAs consisting of (a) circular, (b) square, (c) hexagonal, triangular lenses.

for the rectification of these aberrations. In order to illustrate the large image quality deterioration resulting from even slight misalignment we used the method in [5] to visualize a $2 \mathrm{D}$ misrotated and corrected image. The results of this process for a frontal 2D view are depicted in Fig. 3.

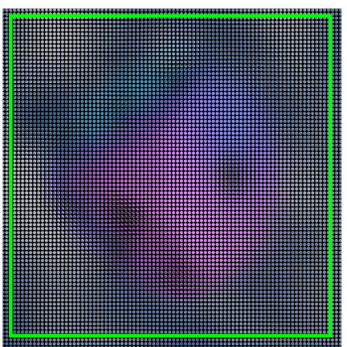

(a)

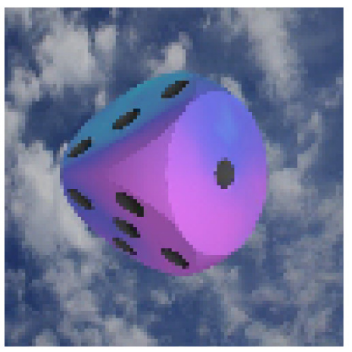

(c)

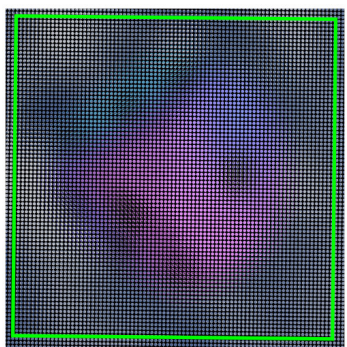

(b)

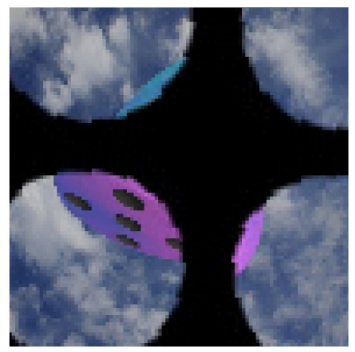

(d)
Fig. 3. (a) Correctly rotated InIm using the proposed methodology. (b) Uncorrected InIm acquired with a misrotated LA. The borders in (a), (b) are shown for illustration purposes. (c) Frontal view generated from the InIm in (a). (d) Frontal view generated from the InIm in (b).

The primary goal of the preprocessing stage is the accurate identification of lines forming the grid that identifies the acquired InIm structure. Using these lines accurate segmentation of the formed EIs is possible, and all the acquisition errors can be corrected before propagating in the display setup.

Various methods have been proposed for correcting geometric distortions in LA configurations containing square and hexagonal lenses. All these methods detect and register grid lines and using this metric information transformation matrices are formed that are subsequently applied in the correction procedure. In [7] a method based on the Hough transform was shown that estimated rotation, position and size information for EIs using square lenses. A perspective rectification method also utilizing the Hough transform was shown in [8]. The work in [9] is an improved and statistically based perspective rectification method aimed to square lens InIms. The method proposed in [10] is a generalization of [9] when applied to LAs containing hexagonal lenses.

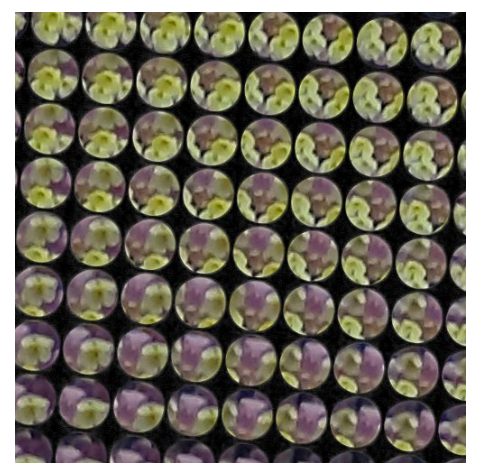

Fig. 4. Acquired InIm containing circular EIs and obvious rotational distortion. The interstitial mask used during the LA construction generates clear dark areas between the circular EIs.

To our best knowledge there are currently no proposed methods for the automatic processing and EI segmentation of InIms captured from LAs with circular lenses. An important characteristic of such setups that we utilize in the presented work is the black area between the circular lenses as shown in Fig. 4.

In particular this area is intentional since during the LA construction process to suppress the unwanted light emitted from the gaps between the circular lens apertures a special interstitial mask is used [11]. The results in an intense contrast difference between the mask and the circular EI content that forms clear circular shapes in the acquired InIm.

To this end we propose a robust processing and segmentation methodology for circular EIs. Our approach is based on the gradient based circular hough transform [12] which is used to extract the center coordinates of a subset of the circular EIs. These coordinates are subsequently fed into a triangulation scheme which generates a part of the distorted InIm grid. By using a statistical approach on the resulting segments we estimate rotation and EI size information and finally reconstruct and overlay an optimal undistorted grid providing transverse as well as longitudinal structure to the underlying EIs. The details of our methodology are outlined in the following sections.

\section{LENS DETECTION}

On a grayscale version of the acquired color InIm we employ a circle detection process that uses the gradient based circular Hough transform. The details of the Hough transform when generalized to circles are elaborated as follows:

The coordinates $(x, y)$ of a point belonging to a circle with radius $r$ and center $\left(a_{1}, a_{2}\right)$ can be written in parametric form 
as

$$
\left\{\begin{array}{l}
t \in[0,2 \pi] \\
x=a_{1}+r \cos (t) \\
y=a_{2}+r \sin (t)
\end{array}\right.
$$

which can be rewritten as:

$$
\left\{\begin{array}{l}
t \in[0,2 \pi] \\
a_{1}=x-r \cos (t) \\
a_{2}=y-r \sin (t)
\end{array}\right.
$$

Because of the above equations and according to [12], it is evident that the Hough transform when extended to circles requires a three-dimensional accumulator space $\mathbf{C}=\left(a_{1}, a_{2}, r\right)$.
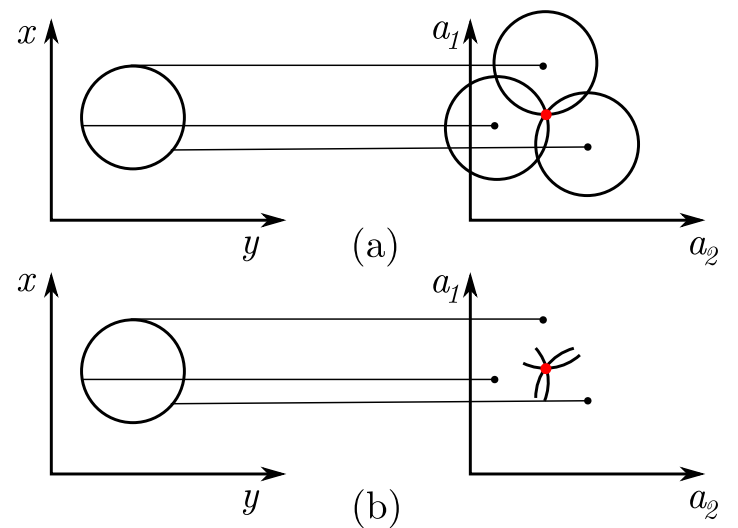

Fig. 5. (a) Each edge pixel (x,y) in the input image (left) is mapped to a circular locus on the accumulator space (right). The intersection point (red) of the circular loci in the accumulator is the center of the detected circle in the input image. (b) The gradient information in the input image shortens the circular loci to arcs.

The classic circular Hough transform operates on the edge version of the input image acquired using the Canny edge detection algorithm. Under known radius $r_{0}$, every edge pixel $(x, y)$ belonging to a circle with center $\left(a_{1}, a_{2}\right)$ in the input image generates a circle with center $(x, y)$ in the two dimensional accumulator space $\left(a_{1}, a_{2}\right)$ as shown in Fig. 5(a).

The intersection point of all the created circular loci in the accumulator space is the center of the detected circle in the input image. This detection approach can be further optimized by noticing that the gradient information existing in the input image is also the gradient of the accumulator space. For a circle $\left(a_{1}, a_{2}, r\right)$ with analytic equation $F$ it is valid that:

$$
\begin{gathered}
F:\left(x-a_{1}\right)^{2}+\left(y-a_{2}\right)^{2}=r^{2} \\
\left\{\begin{array}{l}
\frac{\partial F}{\partial x}=2\left(x-a_{1}\right) \\
\frac{\partial F}{\partial y}=2\left(y-a_{2}\right) \\
\frac{\partial F}{\partial a_{1}}=-2\left(x-a_{1}\right) \\
\frac{\partial F}{\partial a_{2}}=-2\left(y-a_{2}\right)
\end{array}\right.
\end{gathered}
$$

The gradient vector is always pointing towards the detected circle centre. Therefore by using a small angle tolerance for every point of the gradient operator, the circular loci of the accumulator space are limited to intersecting arcs shown in Fig. 5(b). This makes the location of the intersection much more computationally efficient with an added reduction on false detections.

Therefore the circle detection approach we followed can be outlined as follows:

- Calculate the gradient vector and its direction for all pixels $(x, y)$ of the input image.

- Apply Canny edge detection on the input image.

- For every edge pixel register arcs on the accumulator space using the gradient angle information.

- Locate the intersections forming peaks in the accumulator space.

This process is repeated for successive discrete values of $r$ contained in an expected interval $\left[r_{\min }, r_{\max }\right]$. We select the value $r_{0}$ corresponding to the accumulator space generating the sharpest peaks. This results in accurately registering circles in the input InIm corresponding to boundaries between the detected EIs and the interstitial mask.

\section{Rotation Angle Estimation}

After completion of the circle detection process the calculated radius of the circular lenses is estimated as $r_{0}$. Therefore

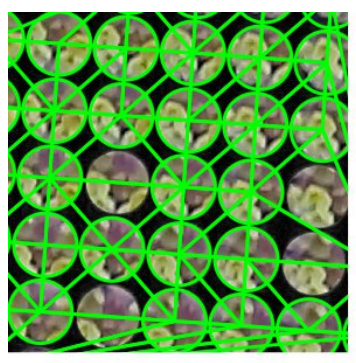

(a)

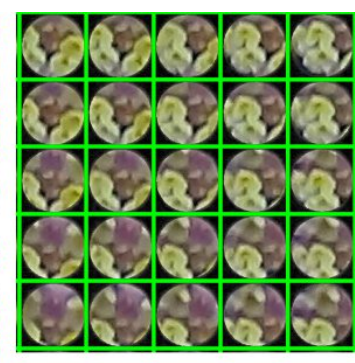

(b)
Fig. 6. (a) Delaunay triangulation on the detected circles centers, (b) The reconstructed and InIm grid that segments the EIs.

we form the sequence

$$
\{[x(i), y(i)]\}, i=1 \ldots N
$$

of the estimated circle centers. These are subsequently used as input to a Delaunay triangulation scheme that connects the centers resulting in a number of $M$ line segments as seen in Fig. 6(a). Each segment is denoted as $s=\left(x_{s}, y_{s}, x_{e}, y_{e}\right)$, where $x_{s}, y_{s}$ are the start point coordinates and $x_{e}, y_{e}$ are the end point coordinates. All these segments form the sequence:

$$
\left\{S_{i}\right\}=\left\{\left[x_{s}(i), y_{s}(i), x_{e}(i), y_{e}(i)\right]\right\}, i=1 \ldots M
$$

Subsequently we calculate the corresponding sequence $\left\{\theta_{i}\right\}$ that contains the angle formed between each segment in $\left\{S_{i}\right\}$ and the $x$-axis. Due to the nature of the utilized triangulation scheme it is expected that in $\left\{\theta_{i}\right\}$ three dominant populations will occur corresponding to segments with ideal angle values of $0^{\circ}, 45^{\circ}, 90^{\circ}$. This is demonstrated in the histogram calculated for $\left\{\theta_{i}\right\}$ that is shown in Fig. 7.

By using a tolerance of $20^{\circ}$ we isolate the intervals $\left[-20^{\circ}, 20^{\circ}\right]$ and $\left[70^{\circ}, 110^{\circ}\right]$, locate their peak values and keep 


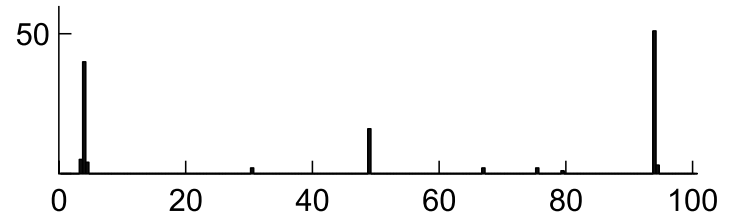

Fig. 7. Histogram for the sequence $\left\{\theta_{i}\right\}$ containing the Delaunay segment angles.

the segments inside a $1^{\circ}$ around them. These peaks correspond to the ideal edges of $0^{\circ}, 90^{\circ}$ in the distorted InIm grid.

Therefore we form the corresponding horizontal and vertical segment populations denoted as $S_{h}, S_{v}$, we calculate the values

$$
\begin{aligned}
x_{h} & =\frac{\operatorname{mean}\left(\Delta x\left\{S_{h}\right\}\right)+\operatorname{mean}\left(\Delta y\left\{S_{v}\right\}\right)}{2} \\
y_{h} & =\frac{\operatorname{mean}\left(\Delta y\left\{S_{h}\right\}\right)+\operatorname{mean}\left(\Delta x\left\{S_{v}\right\}\right)}{2}
\end{aligned}
$$

and we finally calculate the optimal rotation matrix $R$ as:

$$
R=\left(\begin{array}{cc}
\cos \theta & \sin \theta \\
-\sin \theta & \cos \theta
\end{array}\right)=-\frac{1}{\sqrt{x_{h}^{2}+y_{h}^{2}}}\left(\begin{array}{cc}
x_{h} & y_{h} \\
-y_{h} & x_{h}
\end{array}\right)
$$

After $R$ is applied we use the following method in order to segment the EIs by identifying individual EI rows and columns as shown in Fig. 6(b).

\section{GRID RECONSTRUCTION}

Following the estimation of $\theta$, a hierarchical clustering procedure [13] is applied twice in order to assign the detected center coordinates in columns and rows.

From the sequence of centers in (5) we use their $x$ coordinates and form $N_{V}$ vertical classes (columns). These are subsequently sorted and relabeled according to their mean value and they are defined by the following equations:

$$
\begin{gathered}
X_{v}(j): v=1,2, \ldots N_{X} \quad j=1,2, \ldots N_{v} \\
\left|X_{v}(j)-X_{v}\left(j^{\prime}\right)\right|<r_{0} \forall j, j^{\prime} \\
X_{v}(j)<X_{v^{\prime}}\left(j^{\prime}\right) \Rightarrow v<v^{\prime} \forall j, j^{\prime}
\end{gathered}
$$

where $v$ denotes the index of a class in a population of $N_{X}$ formed classes, $j$ denotes the index of an element inside the class and $r_{0}$ is the radius used as the clustering threshold. It is obvious that:

$$
\sum_{v=1}^{N_{X}} N_{v}=N
$$

Similarly and using the same clustering threshold, a number of $M_{Y}$ horizontal classes (rows) are formed containing only $y$-coordinates, and they are also relabeled according to their mean value:

$$
\begin{gathered}
Y_{h}(j): h=1,2, \ldots M_{Y} \quad j=1,2, \ldots M_{h} \\
\left|Y_{h}(j)-Y_{h}\left(j^{\prime}\right)\right|<r_{0} \forall j, j^{\prime} \\
Y_{h}(j)<Y_{h^{\prime}}\left(j^{\prime}\right) \Rightarrow h<h^{\prime} \forall j, j^{\prime}
\end{gathered}
$$

$$
\sum_{h=1}^{M_{Y}} M_{h}=N
$$

Subsequently we evaluate the parameters $x_{0}, y_{0}, h$ to fit a grid of equidistant vertical and horizontal lines to the formed classes. Using the columns shown in Fig. 8 we form the

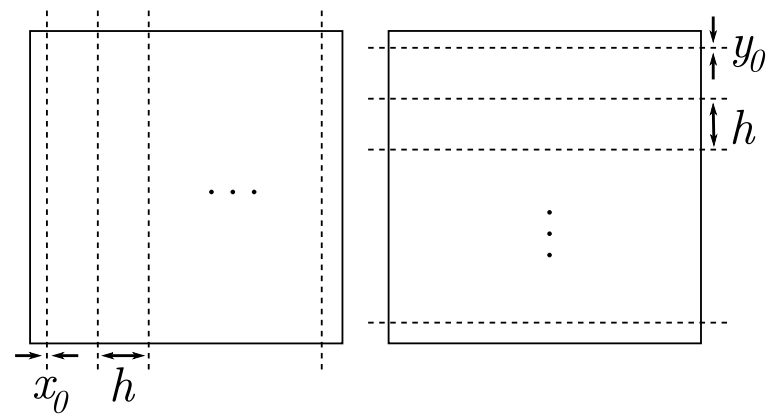

Fig. 8. Registration of a grid with interline distance $h$ starting at $\left(x_{0}, y_{0}\right)$.

following equations that consist of an overdetermined $2 N \times 3$ linear system (OLS):

$$
\begin{gathered}
x_{0}+1 \cdot h=X_{1}(j), j=1,2, \ldots N_{1} \\
x_{0}+2 \cdot h=X_{2}(j), j=1,2, \ldots N_{2} \\
\vdots \\
x_{0}+N_{X} \cdot h=X_{N_{X}}(j), j=1,2, \ldots N_{N_{X}}
\end{gathered}
$$

Using the rows we also form the corresponding OLS:

$$
\begin{gathered}
y_{0}+1 \cdot h=Y_{1}(j), j=1,2, \ldots M_{1} \\
y_{0}+2 \cdot h=Y_{2}(j), j=1,2, \ldots M_{2} \\
\vdots \\
y_{0}+M_{Y} \cdot h=Y_{M_{Y}}(j), j=1,2, \ldots M_{M_{Y}}
\end{gathered}
$$

These two systems are combined and expressed in vector form as:

$$
\begin{gathered}
\mathbf{Q}\left(\begin{array}{c}
x_{0} \\
y_{0} \\
h
\end{array}\right)=\mathbf{b} \\
\mathbf{Q}=\left(\begin{array}{ccc}
\mathbf{0} & \mathbf{1} & \mathbf{V e c}_{\mathbf{1}} \\
\mathbf{1} & \mathbf{0} & \mathbf{V e c}_{\mathbf{2}}
\end{array}\right) \\
\mathbf{b}=\left(\begin{array}{c}
\mathbf{V e c}_{\mathbf{Y}} \\
\mathbf{V e c}_{\mathbf{X}}
\end{array}\right)
\end{gathered}
$$

The vectors of the previous equations are defined as:

$$
\begin{gathered}
\mathbf{V e c}_{1}=\left(\begin{array}{c}
1 \times \operatorname{ones}\left(N_{1}, 1\right) \\
2 \times \operatorname{ones}\left(N_{2}, 1\right) \\
\vdots \\
N_{X} \times \operatorname{ones}\left(N_{N_{X}}, 1\right)
\end{array}\right) \\
\text { Vec }_{2}=\left(\begin{array}{c}
1 \times \operatorname{ones}\left(M_{1}, 1\right) \\
2 \times \operatorname{ones}\left(M_{2}, 1\right) \\
\vdots \\
M_{Y} \times \operatorname{ones}\left(M_{M_{Y}}, 1\right)
\end{array}\right)
\end{gathered}
$$




$$
\begin{gathered}
\mathbf{V e c}_{\mathbf{Y}}=\left(\begin{array}{c}
{\left[X_{1}(j), j=1,2, \ldots N_{1}\right]^{T}} \\
{\left[X_{2}(j), j=1,2, \ldots N_{2}\right]^{T}} \\
\vdots \\
{\left[X_{N_{X}}(j), j=1,2, \ldots N_{N_{X}}\right]^{T}}
\end{array}\right) \\
\mathbf{V e c}_{\mathbf{X}}=\left(\begin{array}{c}
{\left[Y_{1}(j), j=1,2, \ldots M_{1}\right]^{T}} \\
{\left[Y_{2}(j), j=1,2, \ldots M_{2}\right]^{T}} \\
\vdots \\
{\left[Y_{M_{Y}}(j), j=1,2, \ldots M_{M_{Y}}\right]^{T}}
\end{array}\right)
\end{gathered}
$$

Finally the solution is found in a least squares sense by minimizing

$$
\left\|\mathbf{Q}\left(\begin{array}{c}
x_{0} \\
y_{0} \\
h
\end{array}\right)-\mathbf{b}\right\|^{2}
$$

using the pseudoinverse of $\mathbf{Q}$. In order to segment the EIs the calculated grid is shifted by $h / 2$ as shown in Fig 6(b).

\section{EXPERIMENTS, Results AND CONCLUSIONS}

\section{A. Acquired Images}

The described method has been successfully applied on a variety of artificially generated as well as optically acquired images. In this work we demonstrate the evaluation results for one optically acquired and two artificially generated InIms.

The optically acquired InIm is shown in Figs 4, 6 and has been acquired using a Cannon EOS-500D camera using the setup in [9]. It should be noted that lens distortion such as barrel or pincushion has not been considered under the current approach since it is automatically corrected using our camera's firmware. In case the used camera lacks this feature a variety of software approaches can be utilized to remove lens distortion before applying the current framework.

The two artificial InIms namely the "Dice" and the "3D objects" in Fig. 9 were generated using the method described in [14]. Subsequently all the InIms were slightly rotated and the artificial InIms were further contaminated with Gaussian noise of $20,25,30 \mathrm{~dB}$ in order to simulate non-ideal real world capturing conditions.

\section{B. Geometric Consistency Evaluation Measures}

The circular Hough transform introduces some errors to the estimation of the circle centers which in turn affect the calculation of the rotation angle as well as the grid registration process. The reconstructed square grid contains lines that form $0^{\circ}$ or $90^{\circ}$ with the $x$-axis while the detected circle centers present some coordinate perturbation instead of being accurately aligned in the horizontal and vertical directions.

Therefore we introduce the two parameters $\Delta \theta$ and $d$ to estimate the algorithm's consistency. These parameters can be automatically measured on the reconstructed grid. The parameter $\Delta \theta$ refers to the rotation matrix of (9) and measures the angle estimation error in degrees with regards to the ground-truth value. The parameter $d$ is calculated as follows: We calculate both the horizontal as well as the vertical distance of each detected circle center from the reconstructed grid. Ideally these distances should be equal to $h / 2$. These values are collected in the sequence $d$ that is normalized to 1 . Subsequently $d$ is evaluated using its standard deviation $\sigma_{d}$. It must be noted that normalization was applied in order to avoid using pixels as measurement units since the large number of possible LA-CCD configurations offer numerous possibilities in EI resolution.

\section{Results}

In this section we present the geometric parameter evaluation results for the optically acquired InIm image in Fig. 4, as well as the Dice and the 3D objects in Fig. 9. In Figs. 9(a)-(b) we show the corresponding 2D rendered scenes for the "Dice" and the "3D objects". In Figs. 9(c)-(d) we show the acquired and slightly rotated artificial InIm images while in Figs. 9(e)(f) we show the resulting images after correct rotation with the corresponding registered square grid superimposed.

For the InIms in the evaluation set the angle estimation and grid consistency results are shown in Tables I-III. It should be noted that noise levels of $20,25,30 \mathrm{~dB}$ were used only for the evaluation of the artificial InIms as there is no way of assessing the real world noise conditions in an optical pickup procedure. As shown in Tables I-III in the worst case scenario where the

TABLE I

GEOMETRIC CONSISTENCY OF THE REAL INIM

\begin{tabular}{cc}
\hline$\Delta \theta$ & $\sigma_{d}$ \\
\hline $0.26^{\circ}$ & 0.046 \\
\hline
\end{tabular}

TABLE II

GEOMETRIC CONSISTENCY OF THE DICE

\begin{tabular}{lcc}
\hline & $\Delta \theta$ & $\sigma_{d}$ \\
\hline noiseless & $0.04^{\circ}$ & 0.015 \\
\hline $30 \mathrm{~dB}$ & $0.06^{\circ}$ & 0.023 \\
\hline $25 \mathrm{~dB}$ & $0.23^{\circ}$ & 0.035 \\
\hline $20 \mathrm{~dB}$ & $0.37^{\circ}$ & 0.050 \\
\hline
\end{tabular}

TABLE III

GEOMETRIC CONSISTENCY OF THE 3D OBJECTS

\begin{tabular}{lcc}
\hline & $\Delta \theta$ & $\sigma_{d}$ \\
\hline noiseless & $0.03^{\circ}$ & 0.011 \\
\hline $30 \mathrm{~dB}$ & $0.05^{\circ}$ & 0.019 \\
\hline $25 \mathrm{~dB}$ & $0.19^{\circ}$ & 0.022 \\
\hline $20 \mathrm{~dB}$ & $0.28^{\circ}$ & 0.037 \\
\hline
\end{tabular}

evaluated image had high noise levels, the angle estimation error did not exceed $0.37^{\circ}$ and the standard deviation $\sigma_{d}$ did not exceed 0.037 . Therefore the geometric consistency for all the evaluated images remains almost invariant despite the utilized noise levels. Furthermore it is noticed that the geometric evaluation for the optically acquired InIm is in line with the raytraced InIm results. 


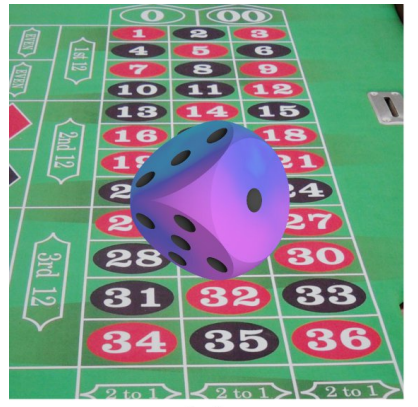

(a)

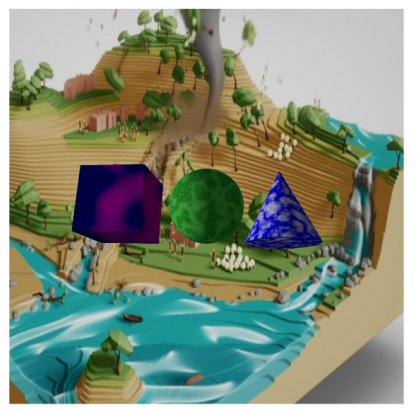

(b)

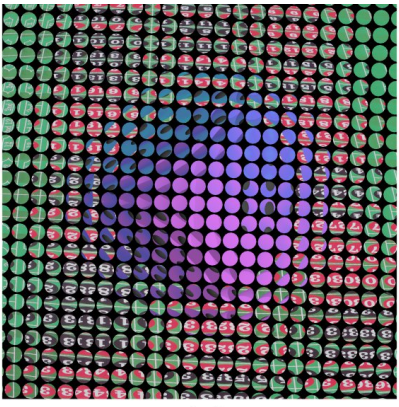

(c)

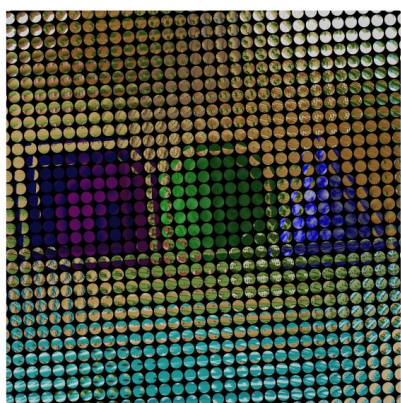

(d)

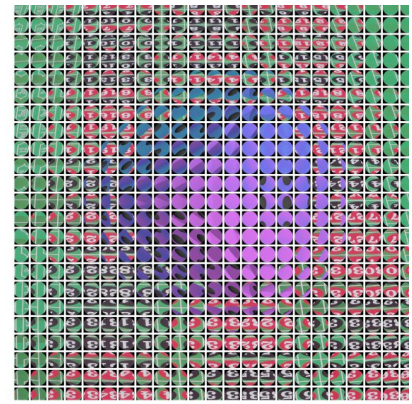

(e)

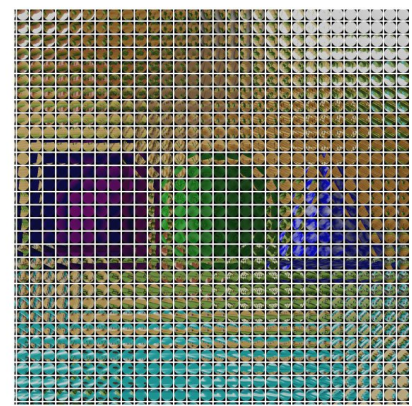

(f)

Fig. 9. Artificial InIms: (a)-(b) are the photographed scenes of a "Dice" and the "3D objects", (c)-(d) are the corresponding slightly rotated InIms, (e)-(f) are the correctly rotated InIms with the registered grid superimposed.

\section{Vi. CONClusions}

In this work we presented a robust methodology for skew angle estimation as well as reconstruction of the grid structure in InIms acquired from circular lens arrays. The presented framework requires a minimal number of parameters and results in the accurate segmentation of the EIs, a process vital to InIm processing tasks.

By utilizing a statistical approach for the skew angle estimation, possible misdetections do not contribute to the estimated angle value. Subsequently by calculating the optimal overlaid grid in a least squares sense we further enhance the method's robustness. The increased robustness of the proposed method is also validated from the fact that the evaluation results are almost invariant despite the use of variable textured scenes and noise levels.

It should be noted that the method was presented for circular LAs with square packing but could easily be augmented for circular LAs with hexagonal packing.

\section{ACKNOWLEDGMENT}

This research has been co-financed by the European Union (European Social Fund) and Greek national funds through the Operational Program "Education and Lifelong Learning" of the National Strategic Reference Framework (NSRF) Research Funding Program: Heracleitus II. "Investing in knowledge society through the European Social Fund."

\section{REFERENCES}

[1] G. Lippmann, "La photographie integràle," Comptes-Rendus Academie des Sciences, vol. 146, pp. 446-451, 1908
[2] J.-H. Park, Y. Kim, J. Kim, S.-W. Min, and B. Lee, "Three-dimensional display scheme based on integral imaging with three-dimensional information processing," Opt. Express, vol. 12, no. 24, pp. 6020-6032, Nov 2004.

[3] J.-S. Jang and B. Javidi, "Formation of orthoscopic three-dimensional real images in direct pickup one-step integral imaging," Optical Engineering, vol. 42, no. 7, pp. 1869-1870, 2003.

[4] G. Passalis, N. Sgouros, S. Athineos, and T. Theoharis, "Enhanced reconstruction of three-dimensional shape and texture from integral photography images," Appl. Opt., vol. 46, no. 22, pp. 5311-5320, Aug 2007.

[5] R. Ng, M. Levoy, M. Brédif, G. Duval, M. Horowitz, and P. Hanrahan, "Light Field Photography with a Hand-Held Plenoptic Camera," Tech. Rep., Apr. 2005.

[6] N. Sgouros, I. Kontaxakis, and M. Sangriotis, "Effect of different traversal schemes in integral image coding," Appl. Opt., vol. 47, no. 19, pp. D28-D37, Jul 2008.

[7] N. P. Sgouros, S. S. Athineos, M. S. Sangriotis, P. G. Papageorgas, and N. G. Theofanous, "Accurate lattice extraction in integral images," Opt. Express, vol. 14, no. 22, pp. 10403-10409, Oct 2006.

[8] K. Hong, J. Hong, J.-H. Jung, J.-H. Park, and B. Lee, "Rectification of elemental image set and extraction of lens lattice by projective image transformation in integral imaging," Opt. Express, vol. 18, no. 11, pp. 12 002-12 016, May 2010.

[9] E. T. Koufogiannis, N. P. Sgouros, and M. S. Sangriotis, "Robust integral image rectification framework using perspective transformation supported by statistical line segment clustering," Appl. Opt., vol. 50, no. 34, pp. H265-H277, Dec 2011.

[10] _ - "Perspective rectification of integral images produced using hexagonal lens arrays," in Intelligent Information Hiding and Multimedia Signal Processing (IIH-MSP), 2012 Eighth International Conference on, july 2012 , pp. 75-78.

[11] R. F. Stevens and T. G. Harvey, "Lens arrays for a three-dimensional imaging system," Journal of Optics A: Pure and Applied Optics, vol. 4 , no. 4 , p. S17, 2002.

[12] C. Kimme, D. Ballard, and J. Sklansky, "Finding circles by an array of accumulators," Commun. ACM, vol. 18, no. 2, pp. 120-122, Feb. 1975.

[13] S. Theodoridis and K. Koutroumbas, Pattern Recognition, Third Edition, 3rd ed. Academic Press, 2006. 
[14] S. S. Athineos, N. P. Sgouros, P. G. Papageorgas, D. E. Maroulis, M. S. Sangriotis, and N. G. Theofanous, "Photorealistic integral photography using a ray-traced model of capturing optics," Journal of Electronic Imaging, vol. 15, no. 4, p. 043007, 2006. 